1 E. Menzel, Rept. Progr. Phys. 26, 47 [1963].

2 W. P. Ellis u. R. L. Schwoebel, Surf. Sci. 11, 82 [1968].

3 G. E. Rhead u. J. Perdereau, in: Colloque International sur la Structure et les Propriétés des Surfaces des Solides, Paris 1969.

4 E. Menzel u. O. Schott, Z. Naturforsch. 20 a, 1221 [1965].

- E. Menzel u. O. Schott, Surf. Sci. 8, 217 [1967]. -

O. Sснотт, Z. Angew. Physik 22, 63 [1967].
5 H. Melle, E. Menzel u. J. Zaunert, Phys. Stat. Sol. (a) 1, 85 [1970].

6 L. Trepte, Chr. Menzel-Kopp u. E. Menzel, Surf. Sci. 8, 223 [1967].

7 Chr. Menzel-Kopp, Z. Naturforsch. 21 a, 1247 [1966].

\title{
Dynamical Interaction of Electron Waves in a Perfect Single Crystal
}

\author{
G. LEHMPFUHL \\ Fritz-Haber-Institut der Max-Planck-Gesellschaft, Berlin-Dahlem \\ (Z. Naturforsch. 27 a, 425-433 [1972]; received 11 November 1971) \\ Dedicated to Prof. Dr. K. Molí̀ne on his 60-th birthday
}

\begin{abstract}
Electron diffraction from a perfect single crystal shows the influence of many-beam interaction. Investigations with a single crystal wedge allow a direct analysis of the Bloch-waves which are displayed into their partial waves. The strength of the dynamical interactions in a $\mathrm{MgO}$ crystal wedge was investigated by comparing the photographic record of a section through the dispersion surface with many-beam calculations. For a direction of the incident beam near the [110] zone axis the interaction of at least 50 beams has to be taken into account. Using a 50-beam calculation the structure potential $V_{111}$ was determined to 1.80 volts $\pm 3 \%$.

The influence of many-beam interactions on the absorption and the extinction fringe profiles in electron micrographs is discussed. Analysis of extinction fringes on the basis of a 50-beam approximation allowed the determination of the mean absorbing potential $V_{0}$ im of $\mathrm{MgO}$ to 0.7 volts $\pm 10 \%$.
\end{abstract}

The electron diffraction pattern from an undistorted perfect single crystal shows very clearly the influence of many-beam interactions. The more accurate the experimental observations become the more accurate the approximations of the dynamical theory have to be, in order to understand and to interpret the diffraction figures. This was already shown in convergent beam investigations ${ }^{1}$ where diffraction diagrams were obtained from parallel sided undistorted crystal regions of only $150 \AA$ to $200 \AA$ in diameter. The probability of finding an undistorted region increases with decreasing diameter of the investigated crystal area. The analysis of the Kossel-Möllenstedt diffraction pattern was done by a many-beam approximation using computer methods. The information from complicated dynamic diffraction diagrams allows an accurate determination of the low-indexed structure potentials and the absorbing potential, provided the applied approximation of the dynamical theory is sufficiently accurate. Low-indexed structure potentials contain information on the binding of the atoms in the crystal. Comparison of accurate experimental

Reprint requests to Dr. G. LehmpFuhl, Fritz-Haber-Institut der Max-Planck-Gesellschaft, D-1000 Berlin 33, Faradayweg $4-6$. observations with calculations for different models are of some interest.

In a perfect crystal with periodic potential the incident plane wave generates wave fields or Blochwaves, which are a superposition of plane waves ${ }^{2}$. The wave vectors of the partial waves in the crystal are connected with the vacuum wave vector by the boundary conditions. These Bloch-waves are separated into different plane waves when the exit surface of the crystal is not parallel to the entrance surface. Consequently, a more direct analysis and observation of the dynamical effects is possible in the diffraction experiment with a crystal wedge.

In the diffraction diagram the strongest partial waves can be observed as a fine structure of the reflections. Their separation is related to the crystal potential. This was shown in many papers for two beam situations in $\mathrm{MgO}^{3}$. Since each fine structure spot represents a point on the dispersion surface, the dynamical interaction of many beams in an undistorted single crystal can be seen very clearly in a photographical record of a section through the dispersion surface ${ }^{4}$. On the other hand each fine-structure spot represents one wave field, containing information on the absorption coefficient of this particular wave field. The absorption coefficients of the 
wave fields are related to the absorbing potential. In a previous paper ${ }^{4}$ we reported on an experiment with a $\mathrm{MgO}$ cube. Now the strength of the dynamical interaction was investigated near a low-indexed zone axis and it will be shown, how the structure potential $V_{111}$ of $\mathrm{MgO}$ can be determined with good accuracy from dynamical interactions of the waves in the crystal.

\section{Experiment}

The experimental method of recording a section through the dispersion surface was described in detail in a previous paper ${ }^{4}$. Therefore the experimental conditions can be described here very briefly. The $\mathrm{MgO}$ crystal wedge of $3-4 \mathrm{~mm}$ edge length was formed by two adjacent (100) cleavage faces. The crystal wedge with a wedge angle of $90^{\circ}$ has the thin region only very near the edge. It is possible to find undistorted regions along the edge of several microns length. The orientation of the crystal was adjusted so that the 002 interference was excited. Figure 1 shows the principle

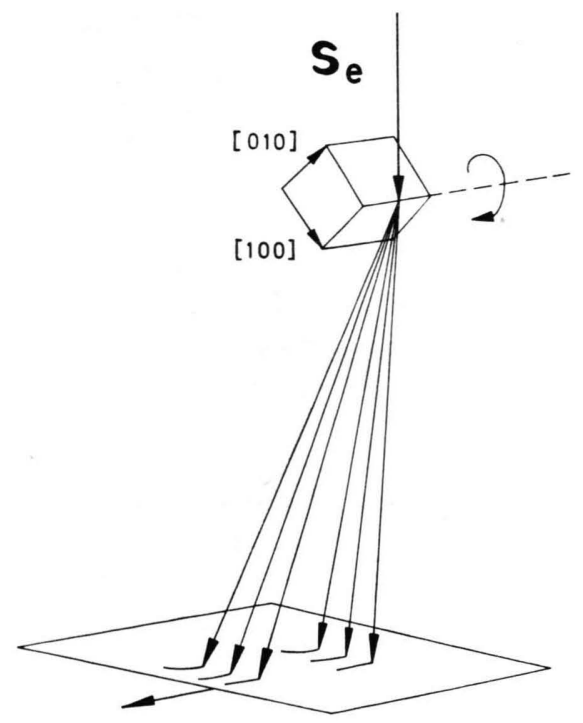

Fig. 1. Principle of recording a section through the dispersion surface. While the crystal is rotated about the edge (parallel to $[001])$ the photographic plate is moved in the direction of the arrow. Each of the two reflections is separated into three partial waves. $\mathrm{S}_{\mathrm{e}}$ is the direction of the incident beam.

for recording a section through the dispersion surface. While rotating the crystal about the [001]-axis which was parallel to the edge, the photographic plate was moved in the direction of the arrow. The diffraction situation can be described exactly by the two parameters $G$ and $H$ as shown in Fig. 2 for a given wavelength of the electrons. We see the intersection circle of the Ewald-sphere with the (110) plane of the reci- procal lattice. The Ewald-sphere intersects the [110]axis at $G$ and the [001]-axis at $H$. In the following we use for $G$ as units the length of the vector [110] and for $H$ the length of the vector [001] of the reciprocal lattice. That means e. g. if $G=4$ and $H=2$, the Ewald-

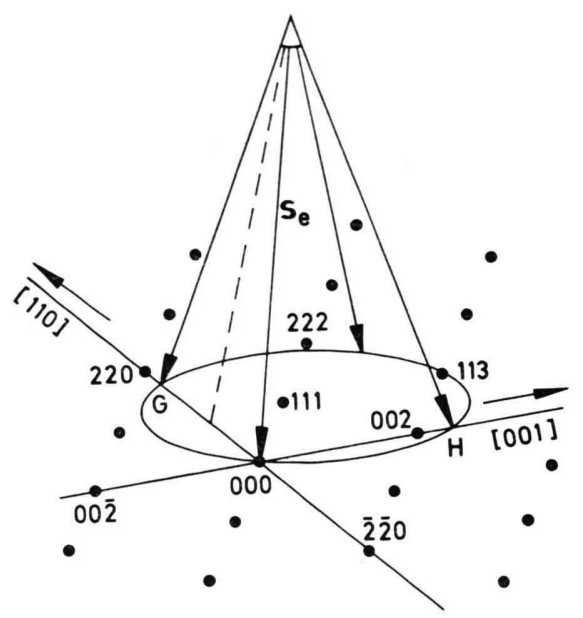

Fig. 2. Section of the Ewald sphere with the (110) plane of the reciprocal lattice. The diffraction situation is defined by the intersection points $G$ and $H$ of the Ewald sphere with the [110] axis and the [001] axis. $S_{e}$ is the direction of the incident beam.

sphere intersects the [110]-axis in the point 440 of the reciprocal lattice and the [001]-axis in the point 002 . If $H=2$ the 002 interference is exactly excited. Rotating the crystal about the [001]-axis for recording a section through the dispersion surface, the excitation conditions for 002 remain constant while $G$ changes. Such a section through the dispersion surface is shown in Fig. $3 \mathrm{a}$. The influence of accidental interactions can clearly be seen in this diagram. Near the zone axis [110] at $G=0$ we see three strong branches of the dispersion surface and a weak fourth one. At $G=2$ the reflections 220 and 222 are exactly excited. We see the strong dynamical interaction in the strongly deformed branches of the dispersion surface. A 19-beam calculation in Fig. $3 \mathrm{~b}$ was in good qualitative agreement with the experimental observations. The calculations were done as described in Refs. ${ }^{4}$ or ${ }^{5}$. The absorption was taken into account by an imaginary potential (absorption potential) using a perturbation method of KAMBE ${ }^{6}$. In the diagram only the branches of the dispersion surface are plotted belonging to partial waves with sufficient intensity.

\section{Strength of the Dynamical Interaction}

A more thorough comparison of calculation with experimental observations shows disagreement, both in the separation of the branches of the dispersion

* Figures 3, 4 and 5 on p. $428 \mathrm{a}, \mathrm{b}$. 
surface and the intensities of the partial waves. The separation of the branches of the dispersion surface depends on the position in the reciprocal space. It is very difficult to determine exactly the plane of intersection with the dispersion surface from an experiment. In Fig. 4 we see how sensitively the section through the dispersion surface changes with the excitation eror of the 002 interference. The sections are recorded simultaneously in the 220 and in the 222 interference. If 002 is exactly excited the sections should be identical in both reflections because of symmetry. There exist more pairs of such symmetrically related reflections e. g. $11 \bar{T}$ and 113 or $00 \overline{2}$ and 004 . The observation of the symmetry is a most sensitive test to check if the excitation error of 002 is zero ${ }^{7}$.

For comparison of calculations with experimental observations it is necessary to know exactly the plane of intersection with the dispersion surface. By a sequence of photographical records of sections through the dispersion surface with slightly different excitation errors for 002 one obtains information in a defined volume element of the reciprocal space which can be better compared with many-beam calculations.

Most suitable for an analysis was the distance between the three strong branches of the dispersion surface at $G=0$ and $H=2$ in Fig. 3 and Fig. 5 showing a minimum. This minimum can clearly be seen by rotating the crystal about the [001]-axis while 002 is exactly excited as shown in Fig. 5 a and also by rotating about the [110]-axis in Fig. $5 \mathrm{~b}$ while $G=0$. This characteristic minimum can easily be determined experimentally. So the many-beam calculation need only be done for exact excitation of the 002 interference at $H=2$ and $G=0$ i. e. for the primary beam lying in the (110) plane. The existence of the three strong branches of the dispersion surface indicates strong influence of 111 and $\Pi 11$ reflections.

Firstly it was not possible with a 19-beam calculation to find agreement with observation both in intensity and separation. In Fig. 6 we see the calculated distances between the three strong branches of the dispersion surface for different numbers of beams. Above 50 beams the calculated distances begin to converge. The influence of up to 108 beams was estimated in the following way: Since the program allowed only a 70-beam calculation, the con-

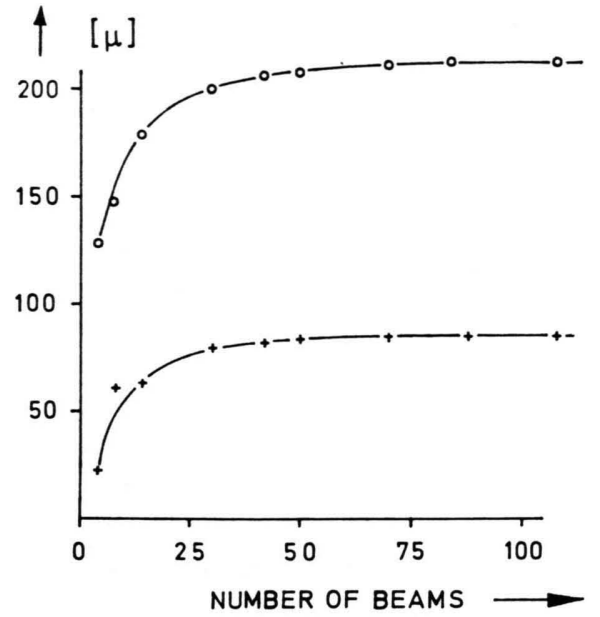

Fig. 6. Calculated distances of the three strong branches of the dispersion surface in the 002 interference at $G=0$ and $H=2$ for different numbers of beams. Energy of the electrons: $50 \mathrm{keV}$.

tribution of the 51-st up to the 70-th beam was estimated from the difference of a 50-beam and a 70beam calculation. Another 68-beam calculation was made including the first 50 beams and the 71-st up to the 88-th beam and finally a 70-beam calculation including the 89-th up to the 108-th beam. The contributions of all these beams were aded giving rise to the value of the distances of the three branches for a 108-beam calculation. It was supposed that this method is allowed because of decoupling of high-order reflections with large excitation errors. This result is in agrement with TinNAPPEL's exact calculations ${ }^{8}$ using group theory in a symmetric case for reducing the fundamental equations of the dynamical theory.

Electron diffraction study of only systematic interactions ${ }^{1}$ showed the importance of many-beam interactions in an one-dimensional diffraction case with strong low-order reflections ${ }^{9}$. In the systematic investigation of $\mathrm{MgO}$, the interaction of 13 beams was taken into acount. Later on it was found that a 7-beam calculation is sufficient for an agreement with the experimental observation. From this result one can estimate that for a full 2-dimensional diffraction situation the interaction of at least 50 beams is important. Also Cowley ${ }^{\mathbf{1 0}}$ and FISHER ${ }^{\mathbf{1 1}}$ have found that in a many-beam calculation near a lowindexed zone axis the interaction of at least 50 beams has to be considered. 


\section{Structure Potential Determination}

The possibility of accurate determination of structure potential from the observation of dynamical many-beam effects in diffraction diagrams is wellknown. So it was investigated how accurate lowindexed structure potentials can be determined from sections through the dispersion surface. In Fig. 7 the sensitivity of the separation of the branches to the structure potential is shown. In the 50-beam calculation only one structure potential was changed about \pm 0.2 Volt at once. We see that all structure potentials have an influence on the separation, the even indexed structure potentials having an influence on both branches which is in the same sense and the odd indexed ones - which depend on the difference of the scattering between oxygen and magnesium-having an opposite influence on the two branches. Furthermore we se that the distances are most sensitive to the structure potential $V_{111}$. Just this low-indexed structure potential is not wellknown, since it is influenced by the binding of the atoms in the crystal that is by the valence electrons.

The question is now, how accurate are the other structure potentials known. For the structure potentials $V_{311}, V_{331}$, and $V_{333}$ such a large variation of 0.2 Volts is not realistic since this would be a change of about $70 \%$. Already Brill, HermanN, and Peters ${ }^{12}$ have shown that the structure factors of $\mathrm{MgO}$ determined from X-ray data for high order indices are in good agreement with the values calculated from tabulated atomic scattering amplitudes. This was confirmed by X-ray investigations of ToGAWA $^{13}$ and SANGER ${ }^{14}$. For values of $\sin \Theta / \lambda$ which
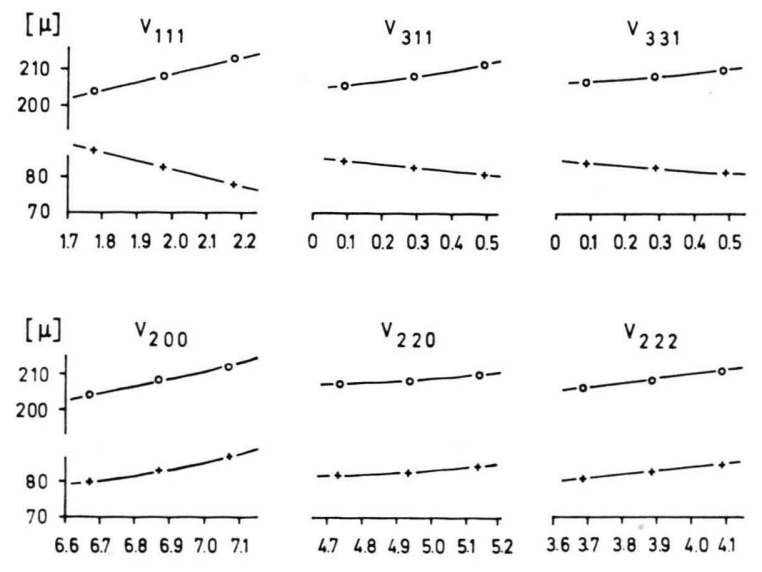

are larger than that corresponding to the 222-reflection the differences in the atomic scattering amplitudes for different quantum chemical models are only in the order of one percent. That means that one is justified to use in the calculation tabulated Hartree-Fock values for the higher indices. The different models of the binding of the atoms in the crystal ${ }^{15,16}$ lead to different scattering amplitudes only for small scattering angles corresponding to low indices. Therefore experimentally determined structure potentials for 002 and 220 were used. The value for 002 was determined with high accuracy of $1 \%$ from convergent beam experiment ${ }^{1}$ taking into account the systematic interaction of 13 beams. This value was confirmed by investigations of HERZBERG ${ }^{17}$. The value of $V_{220}$ was determined from a wedge experiment with an accuracy of $2 \%$ using the 2-nd Bethe approximation considering the influence of many systematic and accidental interactions. In the 2-nd Bethe approximation the influence of other weak excitations is taken into account by additional potentials, so-called "Zusatzpotentiale". HERZBERG ${ }^{17}$ showed that this approximation leads to quite accurate results for exact excitation far away from low-indexed zone axes.

The inaccuracy of all structure potentials used in the calculation lead to very small errors in the calculated distances of the branches of the dispersion surface. So we are justified in determining the structure potential $V_{111}$ by fitting the calculation to the experimental observation.

From Fig. 7 one can see that the Debye-Waller factor had to be known very accurately. The influence of this factor on the separation is shown in

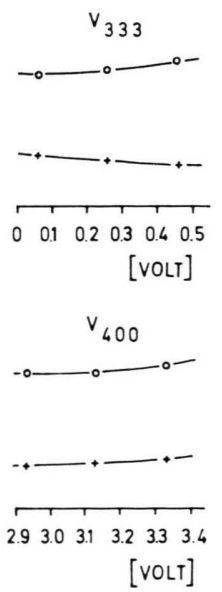

Fig. 7. Distances of the three strong bran. ches of the dispersion surface of $\mathrm{MgO}$ for different structure potentials at $G=0$ and $H=2$ calculated with a 50 -beam approximation for $50 \mathrm{keV}$ electrons. 
G. Lehmpfuhl, Dynamical Interaction of Electron Waves in a Perfect Single Crystal (S. 425).
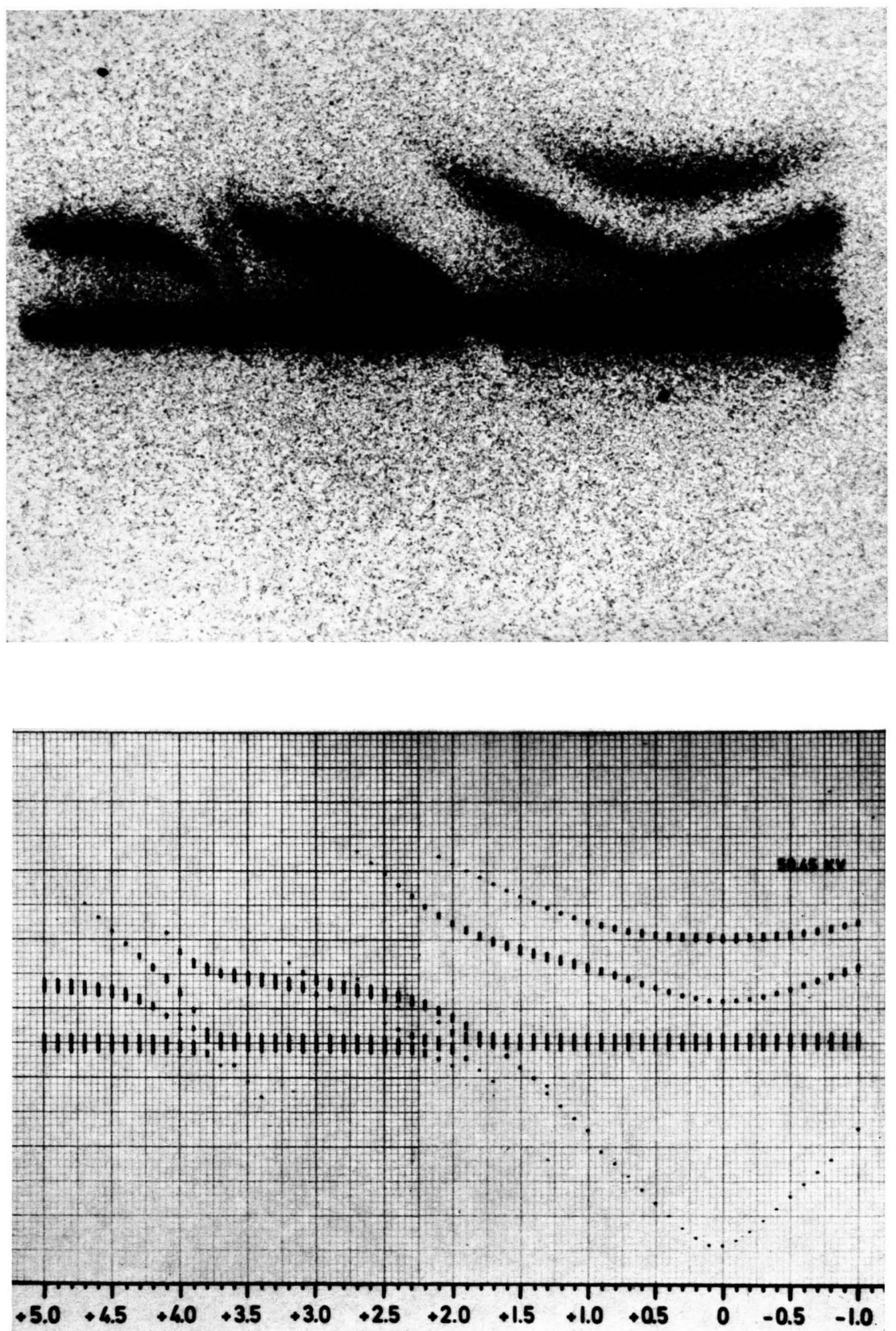

Fig. 3. a) Section through the dispersion surface recorded in the 002 interference of $\mathrm{MgO}$ near the [I110] zone axis with $50.45 \mathrm{kV}$ electrons for $H=2$. The scale indicate the intersection of the Ewald sphere with the [110] axis. b) Calculated section through the dispersion surface, using a 19-beam approximation; length of the rectangles is proportional to the amplitudes. 

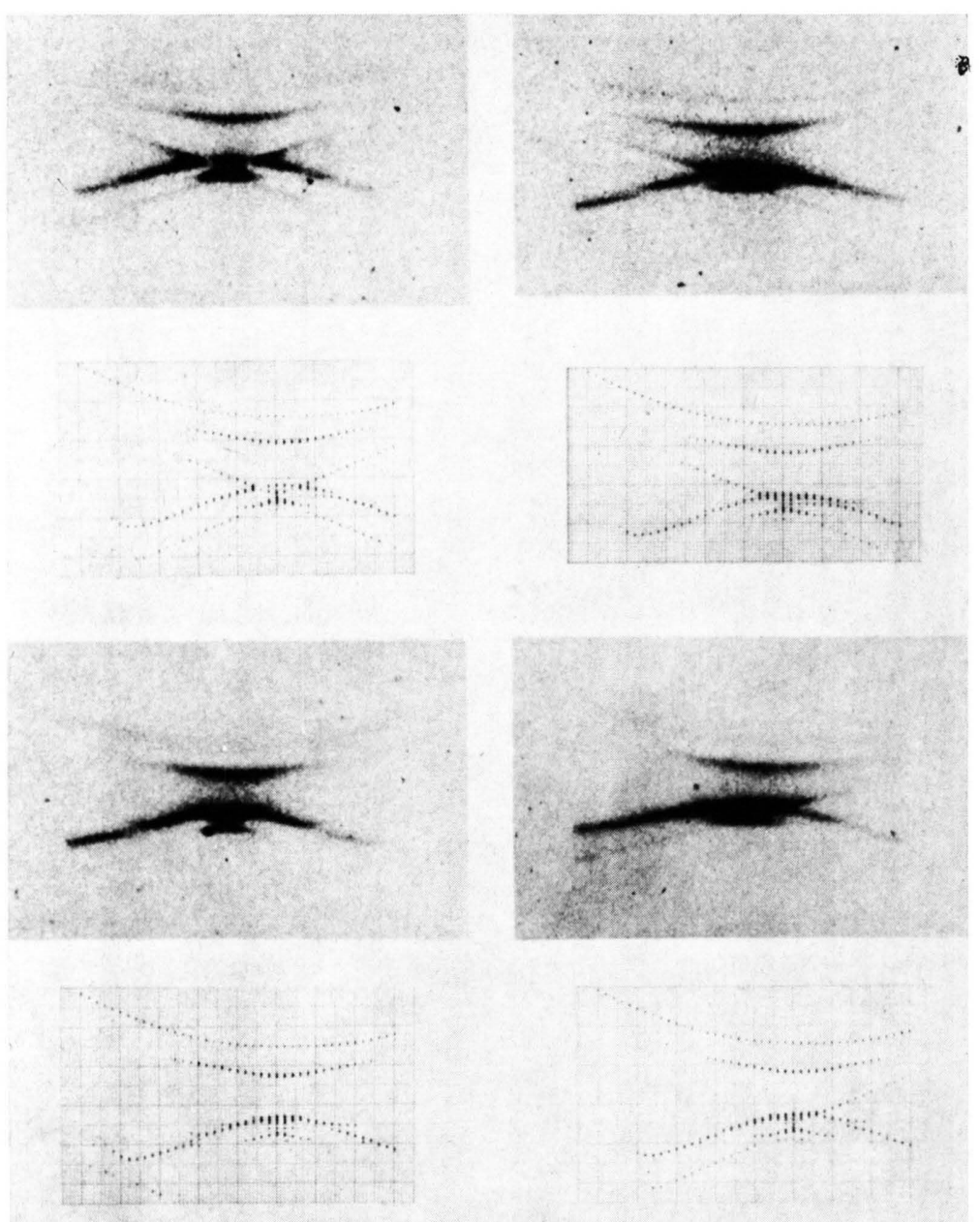

\section{2}

Fig. 4. Section through the dispersion surface recorded from $G=1.0$ to $G=5.0$ in the 220 and in the 222 reflection for two different excitation errors of 002 . For exact excitation of 002 $(H=2)$ the sections should be identical in both reflections (if there would be no absorption).
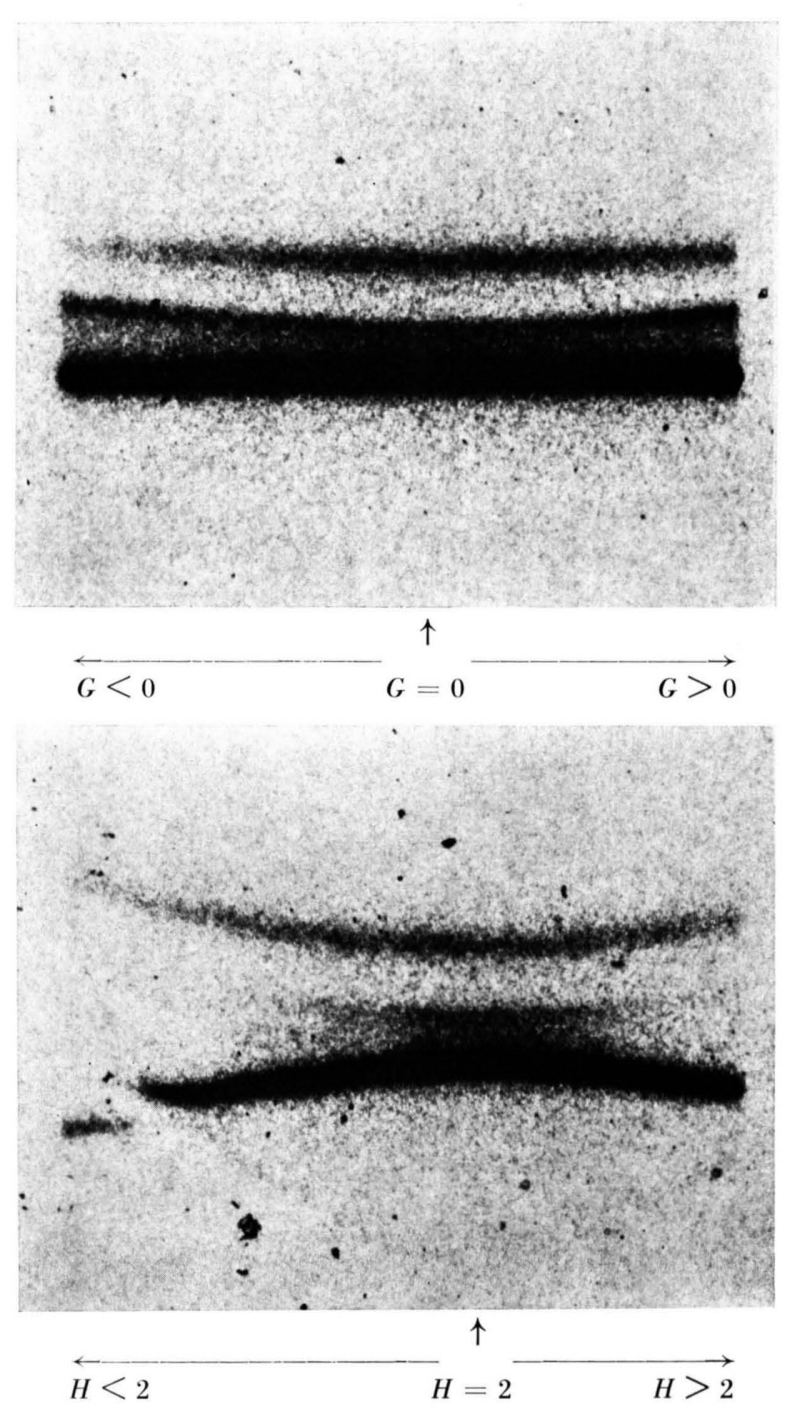

Fig. 5. Sections through the dispersion surface recorded in the 002 reflection showing the minimum distance of the three strong branches at $\mathrm{G}=0$ and $H=2$. a) Crystal was rotated about the [001] axis with $H=2$ from $G=-1$ to $G=+1$; b) The Crystal was rotated about the [110] axis with $G=0$ from $H=+1.5$ to $H=+2.5$. 
Table 1 where the distances are calculated for two different sets of $B$-values. It was not possible to fit the calculated distances of the three strong branches by varying the $V_{111}$ structure potential using the $B$-value of TOGAWA ${ }^{13}$ or the values of SANGER ${ }^{14}$

Table 1. Calculated distances of the three strong branches of the dispersion surface of $\mathrm{MgO}$ for $50 \mathrm{keV}$ electrons at $G=0$ and exact excitation of 002 for different coefficients $B$ of the Debye-Waller factor (Camera length $405 \mathrm{~mm}$ ).

\begin{tabular}{llll}
\hline \multicolumn{2}{c}{ Distances } & Temperature factor & References \\
\hline $83.3 \mu$ & $210 \mu$ & $\begin{array}{l}B_{\mathrm{Mg}}=0.26 \AA \\
B_{0}=0.19 \AA\end{array}$ & TOGAWA $^{13}$ \\
$81.1 \mu$ & $208 \mu$ & $\begin{array}{l}B_{\mathrm{Mg}}=0.30 \AA \\
B_{\mathrm{O}}=0.30 \AA\end{array}$ & GOODMAN $^{18}$ \\
& & &
\end{tabular}

$\left(B_{0}=0.315 \AA^{2}\right.$ and $\left.B_{\mathrm{Mg}}=0.346 \AA^{2}\right)$. With the $B$ value of Goodman ${ }^{18}$ which was obtained for $\mathrm{MgO}$ from electron diffraction experiment the structure potential $V_{111}$ could be determined with about $3 \%$ accuracy. In Table 2 the result is shown together

Table 2. Low-indexed structure potentials which were used in the many-beam calculation with temperature factor $B=0.3 \AA^{2}$ (l. c. Ref. ${ }^{18}$ ). The value for 111 was determined in the present experiment.

\begin{tabular}{lllll}
\hline$h k l$ & $\begin{array}{c}V_{h k l}\left(T=300^{\circ} \mathrm{K}\right) \\
\text { Volt }\end{array}$ & $\begin{array}{c}V_{h k l}\left(T=0^{\circ} \mathrm{K}\right) \\
\text { Volt }\end{array}$ & $\begin{array}{c}\text { Accuracy } \\
\%\end{array}$ & Reference \\
\hline 111 & 1.78 & 1.80 & 3 & $\begin{array}{l}\text { present } \\
\text { experim. }\end{array}$ \\
200 & 6.87 & 6.99 & 1 & 1 \\
220 & 4.92 & 5.10 & 2 & $\mathbf{3}, \mathbf{2 1}$ \\
\hline
\end{tabular}

with the low-indexed structure potentials which were used in the many beam calculation giving the best fit to the experimental observation. All other structure potentials were calculated from relativistic Hartree-Fock electron scattering amplitudes for neutral oxygen and magnesium according to DOYLE and TURNER ${ }^{20}$. The 111 structure potential was determined from the present experiment. This value is in agrement with the value determined in an earlier investigation ${ }^{21}$. The good agreement may be accidental since the separation of the branches of the dispersion surface in a two-beam situation due to the 111 structure potential is very small, so that the error of measurement becomes important. In the zone axis pattern however the additional effect of $V_{111}$ on the separation of the branches can be measured with better accuracy.
With these structure potentials the distances of the three strong branches of the dispersion surface were calculated for different accelerating voltages and in Fig. 8 compared with experimental observation. They show good agreement except near $40 \mathrm{kV}$. The reason may be a distortion of the crystal edge which cannot be detected easily in the diffraction diagram at lower voltages because of the weak intensity or because of the stronger influence of the absorption on the coherent finestructure overlapping at lower voltages.

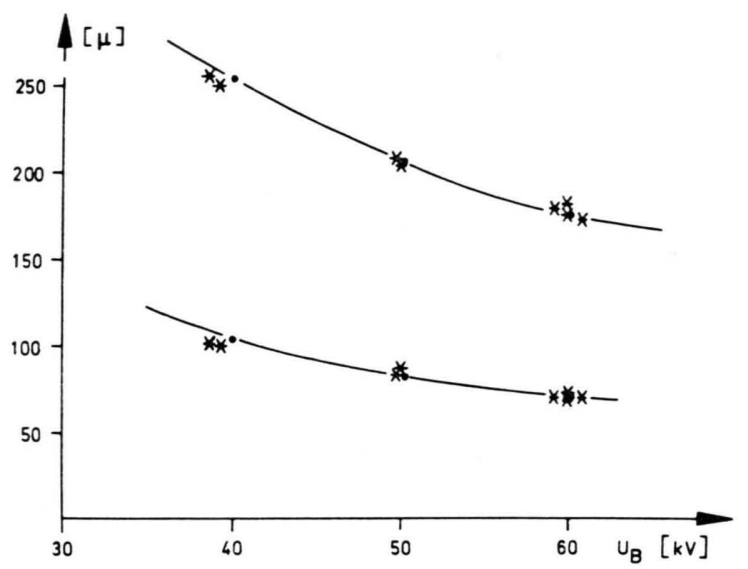

Fig. 8. Distances of the three strong branches of the dispersion surface for different accelerating voltages at $G=0$ and $H=2$. (•) 50-beam calculation with $B=0.3$; (*) experimental observation.

\section{Scattering Factor for Oxygen}

In several papers the ionic state of $\mathrm{MgO}$ and especially the electron state of the oxygen ion was treated ${ }^{22-24}$. It was mentioned above that the lowindexed structure potentials contain information on the binding of the atoms in the lattice. Since the scattering factor of the $\mathrm{Mg}$ ion differs only a little from the scattering factor of the neutral $\mathrm{Mg}$ atom (e. g. Ref. ${ }^{20}$ ) we can recalculate the scattering factor for oxygen in the $\mathrm{MgO}$ lattice from the measured structure potential. From these values the $\mathrm{X}$-ray scattering factors were recalculated and in Fig. 9 compared with scattering factors for ionized oxygen calculated by ToKonAMI ${ }^{24}$ from YAMASHITA's wave functions and with relativistic Hartree-Fock scattering factors ${ }^{20}$ for neutral oxygen. Between the two solid curves there lie the scattering factors of other ionic models of WATOSN ${ }^{15}$ or SUZUKI ${ }^{16}$. Only the scattering factors calculated by FukAMACHI and 


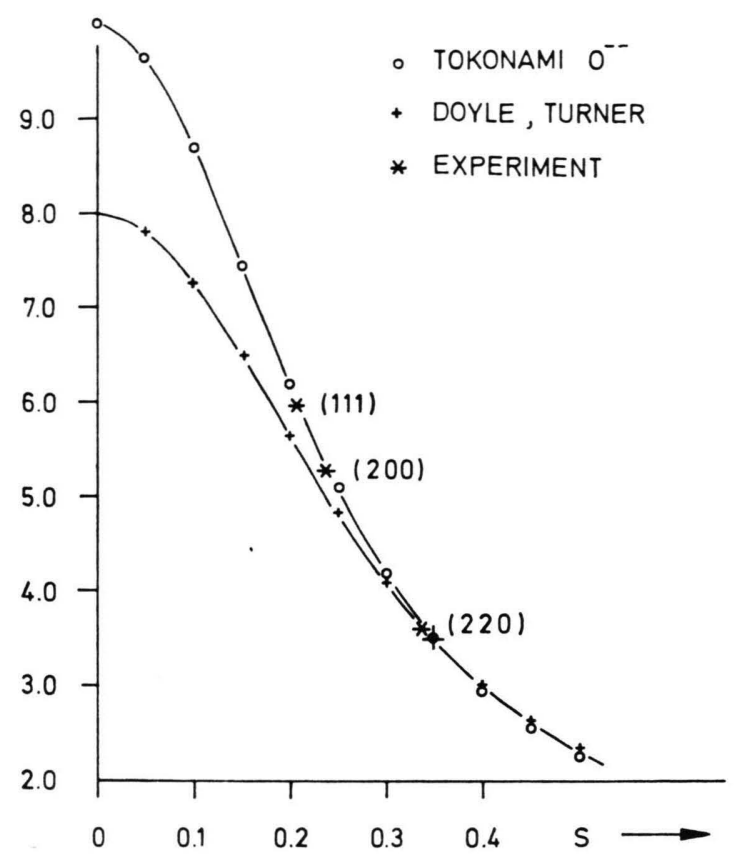

Fig. 9. X-ray scattering factor for oxygen.

Hosoya ${ }^{22}$ are slightly larger than the Tokonami curve. In Fig. 9 the errors of the recalculated scattering factors are different from the errors of the structure potential due to the assumption that the $\mathrm{Mg}$ scattering factors are exactly known ${ }^{25}$. So the error for (111) is only $1 \%$, for (200) $0.5 \%$ and for (220) $2.5 \%$.

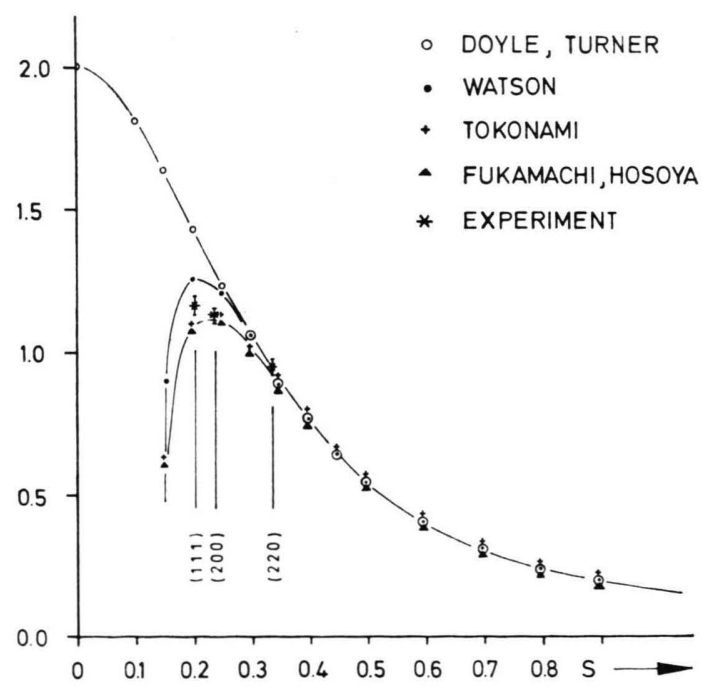

Fig. 10. Electron scattering factor for oxygen.
However the difference between the models is more clearly shown by comparing the scattering factors for electrons $f_{\mathrm{el}}$. These are plotted in Fig. 10, calculated from the X-ray scattering factor using the MotT-relation ${ }^{26}$

$$
f_{\mathrm{el}}=0.023934 \cdot \frac{Z-f_{x}}{s^{2}}
$$

with $s=\sin \Theta / \lambda$ and atomic number $Z$. It is significant that the experimental value for (111) with the error bar lies between the different models and does not fit any of them.

Absorption
The dynamical interaction of the wave fields in
the crystal can have a strong influence on some
phenomena in the diffraction diagram which are
due to the absorption of the electrons. In the sec-
tion through the dispersion surface in Fig. 3 a one
can see that the partial waves belonging to different
branches have different intensities. These differences
are partially due to the absorption and partially due
to many-beam interactions. The influence of the ab-
sorption can be clearly seen by comparison of cal-
culated intensities of the partial waves with experi-
mental observations, and the influence of many-
beam interactions by comparison of a 2 -beam ap-
proximation with many-beam calculations. The am-
plitudes of the partial-waves in the 2 -beam approxi-
mation without absorption are well-known, see e. g.
HASHIMOTO, HowIE, and WHELAN 27 . The two par-
tial waves in the reflection have the same intensity.
In a many-beam approximation without absorption
the partial waves have different intensities. The dif-
ferences increase with increasing dynamical inter-
actions. There exists one partial wave which carries
nearly $25 \%$ of the primary intensity independently
of the dynamical interactions. This partial wave is
represented by the horizontal branch of the disper-
sion surface in Fig. 3 a and b which has only very
few deformations. The other branches are strongly
influenced by the dynamical interactions. The ratio
of the calculated intensities of the two strongest
branches in the 002 reflection for different orienta-
tions is shown in Table 3 . These intensities corre-
spond experimentally to the interpreted intensities
of the finestructure spots. In a two-beam approxima-
tion the ratio is one since the two partial waves of
the reflection have equal intensity. From Table 3
and


one can see that at $G=11.5$ far away from the zone axis the intensity ratio differs only a little from 1 . But near the zone axis the difference due to manybeam interactions is very large. If one would interpret this difference by anomalous absorption one would obtain a Fourier coefficient of the absorbing potential which is too large.

Table 3. Ratio of the intensities of the two strongest branches in the 002 reflection, calculated with a 19-beam approximation without absorption. $G$ indicates as in Fig. 2 the intersection of the Ewald sphere with the reciprocal [110] axis. 002 was exactly excited. $\psi_{002}^{(1,2)}$ are the amplitudes of the two strongest partial waves of the 002 interference.

\begin{tabular}{rlcc}
\hline \multicolumn{1}{c}{$G$} & $I_{002}^{(1)} / I_{002}^{(2)}$ & $\psi_{002}^{(1)}$ & $\psi_{002}^{(2)}$ \\
\hline 2.8 & 0.45 & 0.3235 & -0.4765 \\
5.0 & 0.90 & 0.4433 & -0.4955 \\
11.5 & 0.95 & 0.4873 & -0.4977 \\
\hline
\end{tabular}

GJøNNEs ${ }^{28}$ as well as KAINUMA and YoshiokA ${ }^{29}$ tried to take into acount this many-beam effect in a two-beam approximation by introducing imaginary parts into the structure potentials using complex dynamical potentials. KAINUMA and Yoshioka ${ }^{29}$ called this effect damping due to weak-beam scattering. This approximation was done to estimate the order of magnitude of the influence of many-beam interactions. However with the possibility of using better approximations of the dynamical theory such a treatment becomes unnecessary.

The intensities calculated without absorption do not agree with the experimental observation. This discrepancy and especially the broadening of the different finestructure spots in the diffraction pattern can be explained only by introducing absorption coefficients for the different wave fields ${ }^{30}$ which corresponds to the introduction of an imaginary part of the structure potential due to MOLIÈRE ${ }^{31}$ called absorption potential. v. LAUE ${ }^{32}$ emphasized the fact that each wave field has to be considered as a physical entity with an own characteristic absorption coefficient. This can be understood easily by comparing the electron density distribution of the different wave fields leading to the Borrmann effect in a two-beam situation ${ }^{27}$. In a following paper ${ }^{33}$ this will be shown for a many-beam situation. The absorption coefficients of the wave fields are related to the imaginary part of the structure potential e.g. due to a treatment of $\mathrm{KAMBE}^{6}$. In a two-beam approximation this relation is very simple ${ }^{34}$.

Attempts to replace the introduction of the absorption potential by Hoerni's complex atomic scattering amplitudes by BOERSCH, JESCHKE, and RAITH ${ }^{35}$ cannot explain even for distorted crystals (JESCHKE and NIEDRIG ${ }^{36}$ ) the experimental situation.

\section{Extinction Fringes}

The diffraction pattern is the Fourier transform of the resulting amplitude distribution at the exit surface of the crystal. In a wedge experiment the electron microscopic dark field image of the Pendellösung fringes is in close connection with the finestructure in the diffraction spot. By analysis of Pendellösung fringes one can in principle determine the structure potential from extinction distances (UYEDA and NonOYAma ${ }^{37}$ ) and the absorption potential from the intensity decrease as was shown firstly by Watanabe, Fukuhara, and Kohra ${ }^{38}$. The Fou-
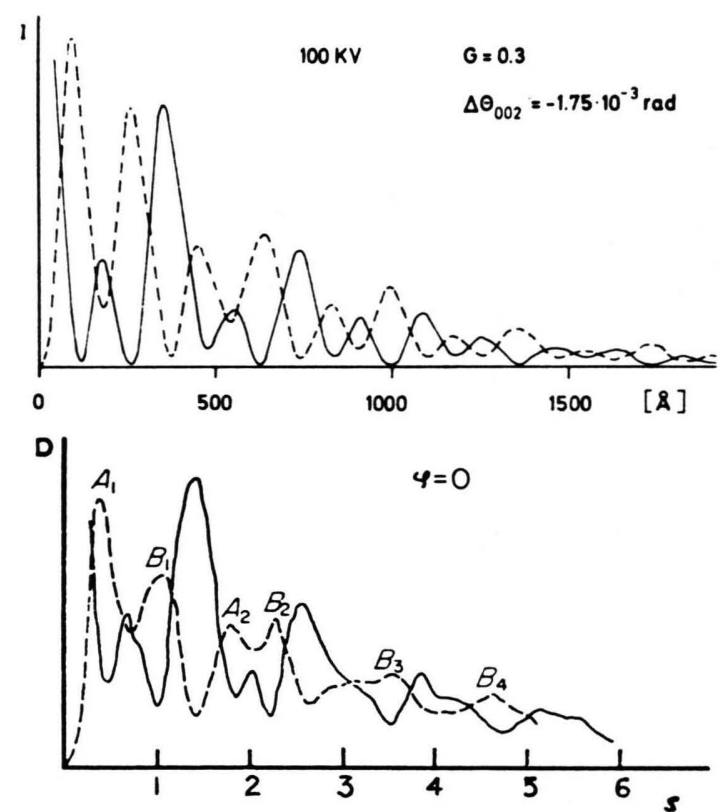

Fig. 11. Extinction fringes of $\mathrm{MgO}$ for $100 \mathrm{keV}$ electrons. 50 -beam calculation near the [I10] zone axis. $\Delta \Theta_{002}$ is the deviation from the Bragg angle of 002. b) Experimental observation of TAKAHASHI ${ }^{40}$.

rier analysis of Pendellösung fringes is very difficult because of the experimental inaccuracies as was shown by Kambe in a private communication. The diffraction pattern however is more suitable for 
such a determination since the strong partial waves can be observed separately. Near a low-indexed zone axis in a many-beam situation the Pendellösung fringes become very complicated as UYEDA ${ }^{39}$ and TAKAHASHI ${ }^{40}$ have shown. In such a situation the extinction distance is no more defined as one can see in Fig. 11 a. From the results above we know that for an analysis of the thickness fringes at least a 50-beam approximation has to be used. The comparison with the 50-beam calculation (Fig. $11 \mathrm{~b})$ shows more details in the thicker region than the experiment. This may be due to an effect of the size of the illuminating aperture ${ }^{1}$ or to inelastic contributions to the micrograph ${ }^{41}$. The difficulties of such an analysis have been discussed ${ }^{42}$. The calculated intensity profiles are sensitive to the structure potentials but not to the Fourier coefficients of the absorbing potential except to the mean absorbing

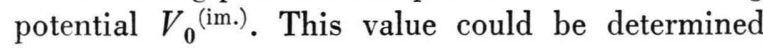
from comparison with the experiment with good accuracy to

$$
V_{0}^{(\mathrm{im} .)}=0.7 \pm 0.07 \text { Volts }
$$

in agreement with measurements of ICHIMIYA ${ }^{43}$ and GOODMAN ${ }^{18}$.

Micrographs of Pendellösung fringes, taken with different reflections may contain informations on the relative phases of the partial waves. This can be seen very clearly in investigations of MAZEL ${ }^{44}$. Dark field micrographs were taken with the weak systematic reflections while 200 was exactly excited. One can see the differences in the micrographs taken with the $\overline{2} 00$ and 400 reflection. The absolute amplitudes of the partial waves in these two reflections are the same under this condition. However the Pendellösung fringes are different because the partial waves must have different phases. In the case of the simple $\mathrm{MgO}$ lattice they have different signs.

1 P. Goodman and G. Lehmpfuhl, Acta Cryst. 22, 14 [1967].

2 H. Bethe, Ann. Phys. Leipzig 87, 55 [1929].

3 H. J. Altenhein and K. Molière, Z. Phys. 139, 103 [1954]. - K. Molière and H. Niehrs, Z. Phys. 140, 58 [1955]; 137, 445 [1954]. - G. Honjo and K. Minama, J. Phys. Soc. Japan 9, 184 [1954]. - J. M. Cowley, P. Goodman, and A. L. G. ReEs, Acta Cryst. 10, 19 [1957] - K. Molière and H. WAgenfeld, Z. Kristallogr. 110, 3 [1958]. Apart from the two-beam situations some threeand four-beam cases have been investigated by MOLIÈrE and WAGENFELD.

4 G. Lehmpfuhl and A. Reissland, Z. Naturforsch. 23 a, 544 [1968].

5 K. Kambe and K. Molière, Advanc. in Structure Research by Diffraction Methods, Vol. 3, Pergamon Press, p. 53.
Already KAMBE ${ }^{45}$ has pointed out the possibility of using many-beam interactions for a determination of phase relations. The observations of dynamical interactions in the diffraction diagram as well as in the micrograph contain in their combination additional information for the measurement of low. indexed Fourier coefficients of the crystal potential and the absorption potential.

\section{Conclusion}

Accurate observations of dynamical situations require the application of higher approximations of the dynamical theory. Generally for such an analysis the crystal surfaces have to be exactly defined. In the case of convergent beam investigations the entrance and exit surfaces have to be completely flat and parallel so that the crystal has uniform thickness. In the wedge experiment the angle between the flat surfaces must be exactly defined. These are very strong conditions which are difficult to fulfil. However if they are fulfilled, electron diffraction is a most suitable tool to determine low-indexed structure potentials from undistorted single crystal areas.

Independent of the crystal shape is the method of WATANABE et al. ${ }^{24}$ in which one observes the relativistic vanishing of a Kikuchi line at a critical accelerating voltage. This results because the effective structure potential of the interference is zero and consequently the extinction length goes to infinity so that the crystal shape has no influence on the observation. It would be of great interest to compare the two methods.

It is a great pleasure to acknowledge Dr. K. KAMBE for many stimulating discussions, Dr. A. W. S. JoHNson for a critical reading of the manuscript and $\mathrm{Mr}$. H. J. Krauss and Mrs. D. Schmidt-Barthmes for assistance with the computation and the experiment.

${ }^{6} \mathrm{~K}$. KAMBE, private communication.

7 The influence of coherent overlapping of the fine-structure spots due to absorption which was first observed by DiD. SZUHN $^{7 \mathrm{a}}$ in a 2 -beam case on the observable symmetry will be discussed in detail shortly.

${ }^{7 a}$ R. Didszunn, Thesis, Freie Universität Berlin 1969, VII. Intern. Congr. on Electr. Microscopy, Grenoble B II, 199 [1970].

8 A. Tinnappel, Thesis, Technische Universität Berlin 1972.

9 Subsequent work of Goodman and Johnson (to be published) showed that normaly the influence of accidental interactions cannot be neglected and that in the case of $\mathrm{MgO}$ only a few directions of incidence exist where one is allowed to consider only systematic interactions.

10 J. M. Cow Ley, Progr. Materials Sci. 13, 269 [1967]. 
11 P. M. J. Fisher, Jap. J. Appl. Phys. 7, 191 [1968].

12 R. Brill, C. HermanN, and Cl. Peters, Z. Anorg. Chem. 257, 151 [1948].

13 S. Togawa, J. Phys. Soc. Japan 20, 742 [1964].

14 P. L. Sanger, Acta Cryst. A 25, 694 [1969].

15 R. E. Watson, Phys. Rev. 111, 1108 [1958].

16 T. Suzuki, Acta Cryst. 13, 279 [1960].

17 B. Herzber G, Z. Naturforsch. 26 a, 1247 [1971].

18 P. Goodman, Acta Cryst. A 27, 140 [1971].

19 G. LehmpfuhL, Z. Kristallogr. 132, 437 [1970].

20 P. A. Doyle and P. S. Turner, Acta Cryst. A 24, 390 [1968].

21 G. LeHMPfuhl and K. Molière, Z. Phys. 166, 389 [1961].

22 T. Fukamachi and S. Hosoya, J. Phys. Soc. Japan [1971].

23 J. Yamashita and S. Asano, J. Phys. Soc. Japan 28, 1143 [1970].

24 M. Tokonami, Acta Cryst. 19, 486 [1965].

25 D. Watanabe, R. Uyeda, and A. Fukuhara, Acta Cryst. A 25, 138 [1969].

26 International Tables III.

27 H. Hashimoto, A. Howie, and M. J. Whelan, Proc. Roy. Soc. London A 269, 80 [1962].

28 J. GJønNEs, Acta Cryst. 15, 703 [1962].
29 Y. KaInuma and H. Yoshioka, J. Phys. Soc. Japan 21, 1352 [1966].

30 H. Niehrs, Phys. Verh. 5, 175 [1954].

31 K. Molı̀̀re, Ann. Phys. Leipzig 34, 461 [1939].

32 M. v. Laue, Acta Cryst. 6, 217 [1953].

33 G. LeHMPFUHL, to be published in Z. Naturforsch.

34 G. Lehmpfuhl and K. Molière, J. Phys. Soc. Japan 17, Suppl. B-II, 130 [1962].

35 H. Boersch, G. JeschKe, and H. RaIth, Z. Phys. 181, 436 [1964].

36 G. Jeschke and H. Niedrig, Acta Cryst. A 26, 114 [1970].

37 R. Uyeda and M. Nonoyama, Jap. J. Appl. Phys. 4, 498 [1965].

38 H. Watanabe, A. Funuhara, and K. Kohra, J. Phys. Soc. Japan 17, Suppl. B-II, 195 [1962].

39 R. Uyeda, Acta Cryst. A 24, 175 [1968].

40 H. Takahashi, Jap. J. Appl. Phys. 8, 296 [1969].

41 Y. Kamiya and R. Uyeda, J. Phys. Soc. Japan 17, Suppl. B-II, 191 [1962].

42 G. Lehmpfuhl, VII. Intern. Congr. on Electr. Microscopy, Grenoble B I, [1970].

43 A. Iснimiya, Jap. J. Appl. Phys. 8, 518 [1969].

44 A. MAzel, J. de Microscopie 11, 207 [1971].

45 K. Kambe, J. Phys. Soc. Japan 12, 13 [1957]. 